University of South Carolina

Scholar Commons

\title{
Elastic Wave Field Computation in Multilayered Nonplanar Solid Structures: A Mesh-Free Semianalytical Approach
}

\author{
Sourav Banerjee \\ University of South Carolina, United States, banerjes@cec.sc.edu \\ Tribikram Kundu
}

Follow this and additional works at: https://scholarcommons.sc.edu/emec_facpub

Part of the Acoustics, Dynamics, and Controls Commons, and the Applied Mechanics Commons

\footnotetext{
Publication Info

Published in The Journal of the Acoustical Society of America, Volume 123, Issue 3, 2008, pages \#1371-. (C)The Journal of the Acoustical Society of America 2008, Acoustical Society of America \& American Institute of Physics Publishing.

Banerjee, S. \& Kundu, T. (2008). Elastic Wave Field Computation in Multilayered Nonplanar Solid Structures: A Mesh-Free Semianalytical Approach. The Journal of the Acoustical Society of America, 123 (3), \#1371. http://dx.doi.org/10.1121/1.2823258
}

This Article is brought to you by the Mechanical Engineering, Department of at Scholar Commons. It has been accepted for inclusion in Faculty Publications by an authorized administrator of Scholar Commons. For more information, please contact digres@mailbox.sc.edu. 


\title{
Elastic wave field computation in multilayered nonplanar solid structures: A mesh-free semianalytical approach
}

\author{
Sourav Banerjee ${ }^{\text {a) }}$ and Tribikram Kundu ${ }^{\text {b) }}$ \\ Department of Civil Engineering and Engineering Mechanics, University of Arizona, Tucson, Arizona 85721
}

(Received 9 August 2007; revised 15 November 2007; accepted 19 November 2007)

\begin{abstract}
Multilayered solid structures made of isotropic, transversely isotropic, or general anisotropic materials are frequently used in aerospace, mechanical, and civil structures. Ultrasonic fields developed in such structures by finite size transducers simulating actual experiments in laboratories or in the field have not been rigorously studied. Several attempts to compute the ultrasonic field inside solid media have been made based on approximate paraxial methods like the classical ray tracing and multi-Gaussian beam models. These approximate methods have several limitations. A new semianalytical method is adopted in this article to model elastic wave field in multilayered solid structures with planar or nonplanar interfaces generated by finite size transducers. A general formulation good for both isotropic and anisotropic solids is presented in this article. A variety of conditions have been incorporated in the formulation including irregularities at the interfaces. The method presented here requires frequency domain displacement and stress Green's functions. Due to the presence of different materials in the problem geometry various elastodynamic Green's functions for different materials are used in the formulation. Expressions of displacement and stress Green's functions for isotropic and anisotropic solids as well as for the fluid media are presented. Computed results are verified by checking the stress and displacement continuity conditions across the interface of two different solids of a bimetal plate and investigating if the results for a corrugated plate with very small corrugation match with the flat plate results.
\end{abstract}

() 2008 Acoustical Society of America. [DOI: 10.1121/1.2823258]

PACS number(s): 43.40.At, 43.20.Fn, 43.20.Bi, 43.20.El [RLW] Pages: 1371-1382

\section{INTRODUCTION}

Elastic wave propagation in a solid medium has been a research topic for over a century. Waves in layered media are comparatively new. Various multilayered structures are being used in layered heat resistant materials, layered insulators, layered cylindrical pipe structures, etc. Ultrasonic, sonic, and other nondestructive evaluations of these materials are needed for assuring integrity of different structural components.

Wave propagation in a layered medium has been a popular problem since mid-19th century. ${ }^{1-4}$ Research activity in this area increased considerably after the frequent use of fiber reinforced composite plates in engineering structures. ${ }^{5-8}$ Irrespective of the type of material layers (isotropic or anisotropic) and field of applications (aerospace, civil, or electronic industries) the integrity testing needs a better understanding of wave propagation in multilayered structures. For a better understanding and interpretation of the experimental results analytical or numerical modeling of the experimental scenario is also very important. Hence, ultrasonic field modeling in multilayered structures has become popular and important to researchers.

A conventional solution of ultrasonic fields in any material generated by transducers is widely known as the ray tracing technique; it involves solution of the Eikonal

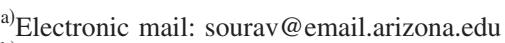

b) Author to whom correspondence should be addressed. Electronic mail: tkundu@email.arizona.edu
}

equation. ${ }^{9}$ Dynamic ray tracing is a major advancement in this field known as the paraxial method or paraxial approximation model. ${ }^{10}$ In the above-mentioned literature the elastic wave propagation in multilayered solids has been analyzed by assuming a plane wave striking the solid. In real-life experiments however, the elastic waves generated by ultrasonic transducers of finite dimension have neither plane nor spherical wave front. No researcher has yet modeled the problem of multilayered solids excited by an ultrasonic beam of finite width generated by a transducer of finite dimension that is placed at a finite distance from the solid. However, this is the real problem geometry for most ultrasonic experiments and needs to be thoroughly investigated.

In this article an efficient mesh-free semianalytical tool called Distributed Point Source Method (DPSM) has been adopted to model the ultrasonic field generated by ultrasonic transducers of finite dimension in multilayered structures when both the structure and the transducers are immersed in a fluid (couplant fluid). Thus, it numerically simulates the ultrasonic experiments for multilayered plate inspection. In the bygone years several researchers have developed various techniques to solve the above-mentioned problems. Some of the most popular methods are finite element method $(\mathrm{FEM}),{ }^{11}$ boundary element method (BEM), ${ }^{12,13}$ multiGaussian beam model (MGBM) ${ }^{14-18}$ charge simulation technique,${ }^{19}$ multiple multipole program (MMP) ${ }^{20-22}$ etc. Although the MGBM technique has some computational advantage over other techniques mentioned here, it also has a number of limitations similar to those of other paraxial models. For example, MGBM cannot correctly model the critical 
reflection phenomenon; it cannot model a transmitted beam at an interface near grazing incidence. This technique also fails if the interface has different curvatures (gradually varying curvature), or when the radius of curvature of the transducer is small, as observed in acoustic microscopy experiments with its tightly focused lens. The multi-Gaussian beam model has not been extended to multilayered structures yet. Therefore, it is important to have a generalized formulation for wave field modeling in multilayered structures without any ray tracing. Such methods should be geometry and material independent. The method should also be capable of handling any geometrical defect or material defect without much difficulty. On the other hand, FEM and BEM packages are very CPU intensive, they require huge amounts of computation memory and time for execution. Similar to MMP another technique was followed by Sanchez-Sesma and Esquivel $^{23}$ to solve SH wave scattering problem for ground motion calculation on alluvial valley. They considered plane wave incidence and formulated the problem in terms of a system of Fredholm integral equations of the first kind with integration paths outside the problem boundary. Similar technique for $\mathrm{P}$ and $\mathrm{SV}$ wave diffraction problem for different surface topographies was reported by Wong ${ }^{24}$ and Dravinski and Mossessian. ${ }^{25}$ However, none of these studies considered any finite dimensional source for elastic waves as done in this article, also they required Green's functions for solid half-spaces and therefore, these techniques cannot be extended to non-half-space problem geometries.

Distributed point source method (DPSM) is a semianalytical technique, which is different from BEM or MMP. DPSM introduces one layer of point sources distributed close to the problem boundary to model the effect of the boundary, and two layers of point sources next to an interface to model the interface effect. Boundary integral equation (BIE) technique and its numerical version, the BEM do not introduce such fictitious sources. However, mathematical justification of introducing artificial point sources to model the boundary effect can be found in the literature on "indirect" BIE (or IBIE and IBEM) ${ }^{26-29}$ The need of introducing point sources for modeling irregular boundaries has been mathematically proven in those publications. In spite of some similarities, it should be pointed out that there are some major differences between DPSM and IBIE. In IBIE, point sources are placed on smooth boundary surfaces. The integral equation thus formed in IBIE has a singular kernel that gives rise to the Fredholm integral equation of the second kind. In the wave propagation literature the IBIE technique has been used to compute the scattered field from an irregular boundary after knowing the incident field in absence of the boundary irregularities. In IBIE formulation the simple incident field is generated by either plane body waves (P, SV, or $\mathrm{SH}$ ) or surface waves-Rayleigh or Love waves. In DPSM, formulation point sources are placed near the boundary and interfaces but slightly outside the domain of interest to avoid singularities. Avoiding the need of solving singular integral equations by moving the points of singularity outside the domain of interest is an advantage of DPSM. Also, in DPSM formulation the boundary and interfaces can have corners and the incident field can be treated as an unknown field, unlike IBIE where the incident field is assumed to be a predefined known field. A bounded beam generated by a transducer of finite dimension is considered as the incident field in the DPSMbased formulation presented here.

The DPSM technique for ultrasonic field modeling was first developed by Placko and Kundu. ${ }^{30}$ They successfully used this technique to model ultrasonic fields in a homogeneous fluid medium. It was then extended to more complicated problems by Banerjee and co-workers such as multilayered fluid structure, ${ }^{31}$ solid half space and plate, with and without anomalies, ${ }^{32-34}$ wave field modeling in solid plates with nonplanar boundaries ${ }^{35}$ and acoustic microscope modeling by Kundu et al. ${ }^{36}$

In the DPSM technique it is necessary to have stress and displacement Green's functions for fluid and solid media. Displacement Green's functions in frequency domain for isotropic solids are available in the literature. ${ }^{37}$ However, explicit expressions for frequency domain stress Green's functions in isotropic material are only available with some approximations. Explicit expressions of displacement and stress Green's functions for isotropic solids without any approximations have been given by Banerjee and Kundu. ${ }^{32}$

A two-layered copper-aluminum bimetal plate is considered for the present study. Ultrasonic fields are calculated in this structure for both planar and nonplanar boundaries for various angles of incidence of bounded ultrasonic beams. Computed results clearly show how the ultrasonic energy in the plate decays with the distance of propagation in flat and corrugated plates.

\section{GENERAL FORMULATION}

A multilayered solid structure is considered. The layers in the multilayered solid can consist of isotropic, transversely isotropic, general anisotropic, or a combination of these materials. In this general formulation any type of anisotropy can be considered and both plane and nonplanar interfaces can be modeled. On two sides of the solid structure Fluids 1 and 2 are used as the coupling fluid that transmits ultrasonic waves from the ultrasonic transducers to the solid structure [see Fig. 1(a)]. To model the ultrasonic field inside the multilayered structure and the fluid, the DPSM technique is employed. Let us consider a multilayered solid structure of $n$ different layers made of $n$ different materials. Hence, there are $n+1$ interfaces say $I_{1}, I_{2}, \ldots, I_{n+1}$. Following the basics of the DPSM technique two sets of point sources are distributed on two sides of every interface making a total of $2(n+1)$ sets of point sources adjacent to $(n+1)$ interfaces, as shown in Fig. 1(a). Each interface acts as a transmitter as well as a reflector of elastic wave energy generated by the ultrasonic transducers. Point sources are also distributed behind the transducer faces. Transducer sources are denoted as $\mathbf{A}_{S}$ and $\mathbf{A}_{R}$ in Fig. 1(a). Two sets of source strength vectors corresponding to the $m$ th interface $I_{m}$ are denoted by $\mathbf{A}_{m}$ (for sources located just above the $m$ th interface) and $\mathbf{A}_{m}^{*}$ (for sources located just below the $m$ th interface). The sources with source strength $\mathbf{A}_{m}$ generate the ultrasonic field in the solid below it and the sources with source strength $\mathbf{A}_{m}^{*}$ generate the ultrasonic field in the solid above it. The total ultrasonic field in each me- 


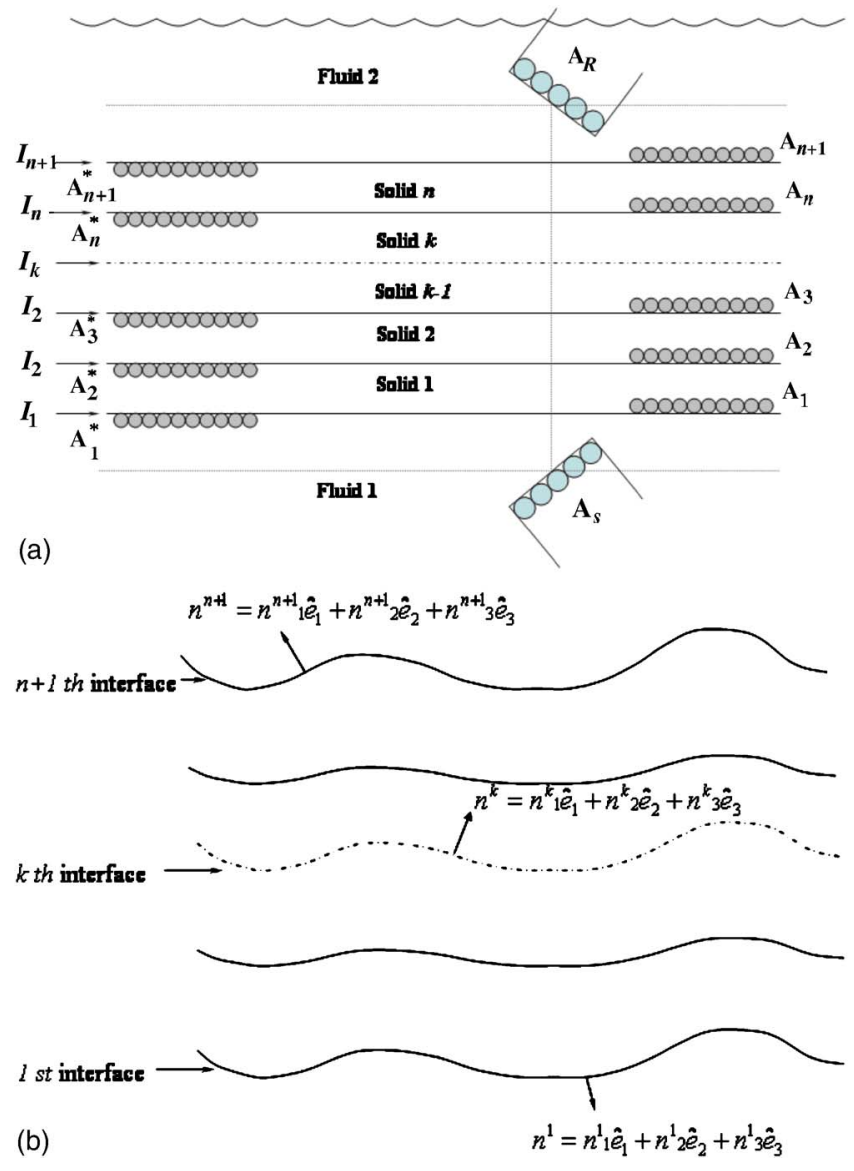

FIG. 1. (Color online) (a) Problem geometry-multilayered solid plate and two ultrasonic transducers immersed in water. Small circles show distributions of point sources, point sources are placed at the centers of these circles. (b) Multilayered solid with nonplanar interfaces and boundaries.

dium is obtained by superimposing the fields generated by two sets of sources. The ultrasonic field generated in Fluid 1 is the summation of fields generated by $\mathbf{A}_{S}$ and $\mathbf{A}_{1}$. The ultrasonic field generated in Solid 1 is the summation of fields generated by $\mathbf{A}_{1}^{*}$ and $\mathbf{A}_{2}$. Similarly the ultrasonic field in Solid 2 is the summation of fields generated by $\mathbf{A}_{2}^{*}$ and $\mathbf{A}_{3}$. The field in Solid $n$ is the summation of fields generated by $\mathbf{A}_{n}^{*}$ and $\mathbf{A}_{n+1}$, and the field in Fluid 2 is obtained from $\mathbf{A}_{n+1}^{*}$ and $\mathbf{A}_{R}$.

\section{A. Matrix formulation}

The particle velocity and pressure in fluids at the fluidsolid interfaces can be expressed in matrix form. ${ }^{18}$ Let $T 1$ and $T 2$ be two different sets of target points in the fluid below and above Interfaces 1 and $n+1$, respectively. The velocity at the target points can be written as

$$
\begin{aligned}
& \mathbf{V}_{T 1}=\mathbf{M}_{(T 1) S} \mathbf{A}_{S}+\mathbf{M}_{(T 1) 1} \mathbf{A}_{1}, \\
& \mathbf{V}_{T 2}=\mathbf{M}_{(T 2) R} \mathbf{A}_{R}+\mathbf{M}_{(T 2) n+1} * \mathbf{A}_{n+1}^{*} .
\end{aligned}
$$

Similarly, the pressure fields at the target points are

$$
\mathbf{P R}_{T 1}=\mathbf{P R}_{T 1}^{s}+\mathbf{P} \mathbf{R}_{T 1}^{1}=\mathbf{Q}_{(T 1) S} \mathbf{A}_{S}+\mathbf{Q}_{(T 1) 1} \mathbf{A}_{1},
$$

$$
\mathbf{P R}_{T 2}=\mathbf{P R}_{T 2}^{s}+\mathbf{P R}_{T 2}^{n+1}{ }^{*}=\mathbf{Q}_{(T 2) R} \mathbf{A}_{R}+\mathbf{Q}_{(T 2) n+1} * \mathbf{A}_{n+1}^{*} .
$$

Elements of the matrices written in Eqs. (1)-(4) are given in Spies. ${ }^{18}$ If the boundary surfaces of the interfaces are nonplanar [Fig. 1(b)], normal stress and normal displacement directions at the interface vary from point to point. The direction cosine of the nonplanar interface at any point on the interface can be defined as $n=\left(n_{1} e_{1}+n_{2} e_{2}+n_{3} e_{3}\right)$. Projections of unit normal $(n)$ on $x_{1}, x_{2}$, and $x_{3}$ axes can be defined according to the problem definition. Point sources needed for modeling solids are different from those used for fluid modeling. Every point source for the solid modeling has three different force components in three mutually perpendicular directions. Stress at point $\mathbf{x}$ generated by a point source acting at point $\mathbf{y}$ in a solid can be obtained from stress Green's functions of that material. For a point source acting at $\mathbf{y}$ in an isotropic solid, the stresses developed at point $\mathbf{x}$ have been given by Banerjee and Kundu. ${ }^{38}$ Displacement Green's functions for transversely isotropic and anisotropic materials have been also presented in the literature. ${ }^{39-42}$ However, as the stress Green's function for anisotropic materials are derivatives of displacement Green's functions those need to be calculated numerically in the absence of any closed form expressions. Assuming a point force acting along the $x_{j}$ direction, stresses at point $\mathbf{x}$ on the boundary of the interface can be denoted by the stress tensor $\boldsymbol{\sigma}^{j}=\sigma_{m n}^{j}$. To obtain stress components that are perpendicular and parallel to a nonplanar interface this stress tensor is to be transformed using the standard stress transformation law $\boldsymbol{\sigma}^{\prime j}=\mathbf{T} \boldsymbol{\sigma}^{j} \mathbf{T}^{T}$, where $\mathbf{T}$ is the transformation matrix. The transformation matrix for point $\mathbf{x}$ on the interface depends on the interface geometry. If the normal to the interface does not have an $x_{3}$ component then $n_{3}=0$, and the matrix $\mathbf{T}$ can be written as

$$
\mathbf{T}=\left[\begin{array}{ccc}
n_{2} & -n_{1} & 0 \\
n_{1} & n_{2} & 0 \\
0 & 0 & 1
\end{array}\right]
$$

To define the boundary conditions at point $\mathbf{x}$, the normal stress perpendicular to the boundary surface and two shear stress components, parallel to the boundary surface are needed. Considering a set of $M$ point sources distributed on the interfaces, the normal stress and the shear stress components can be defined as

$$
\begin{aligned}
S_{22}^{\prime} & =\sum_{m=1}^{M}\left[\left(\sigma_{22}^{\prime}\right)^{m} P_{1}^{m}+\left(\sigma_{22}^{\prime}\right)^{m} P_{2}^{m}+\left(\sigma_{22}^{\prime}\right)^{m} P_{3}^{m}\right] \\
& =\sum_{m=1}^{M} \underline{\mathbf{s}_{22}^{\prime}}(\mathbf{P})^{m}, \\
S_{21}^{\prime} & =\sum_{m=1}^{M}\left[\left(\sigma_{21}^{\prime}\right)^{m} P_{1}^{m}+\left(\sigma_{21}^{\prime}\right)^{m} P_{2}^{m}+\left(\sigma_{21}^{\prime}\right)^{m} P_{3}^{m}\right] \\
& =\sum_{m=1}^{M} \underline{\mathbf{s}_{21}^{\prime} m}(\mathbf{P})^{m},
\end{aligned}
$$




$$
\begin{aligned}
S_{23}^{\prime} & =\sum_{m=1}^{M}\left[\left(\sigma_{23}^{\prime}\right)^{m} P_{1}^{m}+\left({\sigma_{23}^{\prime}}^{2}\right)^{m} P_{2}^{m}+\left(\sigma_{23}^{\prime}\right)^{m} P_{3}^{m}\right] \\
& =\sum_{m=1}^{M} \underline{\mathbf{s}_{23}^{\prime}}(\mathbf{P})^{m}
\end{aligned}
$$

where the definition of $\mathbf{P}$ is given in Banerjee and Kundu. ${ }^{38}$ Displacements at point $\mathbf{x}$ generated by a point source acting at point $\mathbf{y}$ in a solid can be obtained from the displacement
Green's functions. ${ }^{38-42}$ Three displacement components at $\mathbf{x}$ generated by a point force acting along the $x_{j}$ direction are denoted by $G_{1 j}, G_{2 j}$, and $G_{3 j}$. Considering the same point force along the $x_{j}$ direction, the normal displacement of the solid surface at $\mathbf{x}$ can be written as

$$
u_{n}^{j}=G_{1 j} n_{1}+G_{2 j} n_{2}+G_{3 j} n_{3} .
$$

If a set of $M$ point sources are distributed on the interface, then the normal displacement at point $\mathbf{x}$ on the interface can be written as

$$
\underline{u n}=\sum_{m=1}^{M}\left(\begin{array}{l}
\left(G_{11} n_{1}+G_{21} n_{2}+G_{31} n_{3}\right)^{m} P_{1}^{m}+\left(G_{12} n_{1}+G_{22} n_{2}+G_{32} n_{3}\right)^{m} P_{2}^{m}+ \\
\left(G_{13} n_{1}+G_{23} n_{2}+G_{33} n_{3}\right)^{m} P_{3}^{m}
\end{array}\right)=\sum_{m=1}^{M} \underline{\mathbf{G}}^{m} \mathbf{P}^{m} .
$$

Let $T$ be a set of target points in the $m$ th solid layer. Normal displacements at these points $(T)$ on the interface can be written in the following form:

$$
\underline{\mathbf{u n}}_{T}=\mathbf{D S n} \mathbf{n}_{T m} * \mathbf{A}_{m}^{*}+\mathbf{D S n} \mathbf{n}_{T m+1} \mathbf{A}_{m+1} .
$$

Similarly transformed normal stress and shear stresses at the target points $(T)$ at the $m$ th solid layer can be written as

$$
\begin{aligned}
& \mathbf{s 2 2}_{T}^{\prime}=\mathbf{S 2 2}_{T m}^{\prime} * \mathbf{A}_{m}^{*}+\mathbf{S} 22_{T m+1}^{\prime} \mathbf{A}_{m+1}, \\
& \mathbf{s 2 1}_{T}^{\prime}=\mathbf{S} 21_{T m}^{\prime} * \mathbf{A}_{m}^{*}+\mathbf{S} 2 \mathbf{1}_{T m+1}^{\prime} \mathbf{A}_{m+1}, \\
& \mathbf{S 2 3}_{T}^{\prime}=\mathbf{S} 23_{T m}^{\prime} * \mathbf{A}_{m}^{*}+\mathbf{S} 2 \mathbf{3}_{T m+1}^{\prime} \mathbf{A}_{m+1} .
\end{aligned}
$$

Matrices $\mathbf{D S n} \mathbf{n}_{T S}, \mathbf{S 2 2}{ }_{T S}^{\prime}, \mathbf{S} 21_{T S}^{\prime}$, and $\mathbf{S} 23_{T S}^{\prime}$ are given in Banerjee and Kundu. ${ }^{38}$ Subscripts $T$ and $S$ denote sets of target and source points, respectively. The displacement components at point $\mathbf{x}$ generated by a point source at $\mathbf{y}$ are also available in the literature ${ }^{38}$ and are not repeated here.

For a nonplanar corrugated interface using the direction cosines $\left(n_{i}\right)$ of the normal vector to the interface, the displacement component normal to the corrugated interface at point $\mathbf{x}$ can be written as

$$
u_{f n}=u_{1} n_{1}+u_{2} n_{2}+u_{3} n_{3}
$$

Therefore, in presence of transducers (see Fig. 1) the displacement of the fluid at Interfaces 1 and $n+1$ can be written as

$$
\begin{aligned}
\underline{\mathbf{U n}}_{I 1}= & \left(\left(\mathbf{D F} 3_{(I 1) S}\right) n_{3}+\left(\mathbf{D F 2} \mathbf{2}_{(I 1) S}\right) n_{2}+\left(\mathbf{D F} \mathbf{1}_{(I 1) S}\right) n_{1}\right) \mathbf{A}_{S} \\
& +\left(\left(\mathbf{D F 3} \mathbf{3}_{(I 1) 1}\right) n_{3}+\left(\mathbf{D F 2} \mathbf{2}_{(I 1) 1}\right) n_{2}\right. \\
& \left.+\left(\mathbf{D F 1} \mathbf{1}_{(I 1) 1}\right) n_{1}\right) \mathbf{A}_{1},
\end{aligned}
$$

$$
\begin{aligned}
\underline{\mathbf{U n}}_{I n+1}= & \left(\left(\mathbf{D F 3} \mathbf{3}_{(I n+1) R}\right) n_{3}+\left(\mathbf{D F 2} \mathbf{I}_{(I n+1) R}\right) n_{2}\right. \\
& \left.+\left(\mathbf{D F} \mathbf{1}_{(I n+1) R}\right) n_{1}\right) \mathbf{A}_{R}+\left(\left(\mathbf{D F} 3_{(I n+1) n+1} * n_{3}\right.\right. \\
& \left.+\left(\mathbf{D F 2} 2_{(I n+1) n+1} *\right) n_{2}+\left(\mathbf{D F} \mathbf{1}_{(I n+1) n+1} *\right) n_{1}\right) \mathbf{A}_{n+1}^{*}
\end{aligned}
$$

or,

$$
\begin{aligned}
& \underline{\mathbf{U n}}_{I 1}=\mathbf{D F} \mathbf{n}_{(I 1) S} \mathbf{A}_{S}+\mathbf{D F n}_{(I 1) 1} \mathbf{A}_{1}, \\
& \underline{\mathbf{U n}}_{I n+1}=\mathbf{D F} \mathbf{n}_{(I n+1) R} \mathbf{A}_{R}+\mathbf{D F} \mathbf{n}_{(I n+1) n+1} * \mathbf{A}_{n+1}^{*} .
\end{aligned}
$$

Matrix $\mathbf{D F} \mathbf{n}_{T S}$ is given in Banerjee and Kundu. ${ }^{38}$ Let us consider a set of target points on "Interface $k$ " that is denoted as $I k$. The transformed normal stress and shear stress matrices for the referenced target points can be written as

$$
\begin{aligned}
& \mathbf{S 2 2}_{I k}^{\prime}=\mathbf{S 2 2}_{I k k}^{\prime} \mathbf{A}_{k}^{*}+\mathbf{S} 22_{I k(k+1)}^{\prime} \mathbf{A}_{k+1}, \\
& \mathbf{S 2 1}_{I k}^{\prime}=\mathbf{S 2 1}_{I k k^{*}}^{\prime} \mathbf{A}_{k}^{*}+\mathbf{S} 21_{I k(k+1)}^{\prime} \mathbf{A}_{k+1}, \\
& \mathbf{S 2 3}_{I k}^{\prime}=\mathbf{S 2 3}_{I k k^{*}}^{\prime} \mathbf{A}_{k}^{*}+\mathbf{S} 23_{I k(k+1)}^{\prime} \mathbf{A}_{k+1} .
\end{aligned}
$$

Similarly on Interface $k+1$ the set of target points are denoted as $I k+1$ and the transformed normal and shear stresses on the interface can be written as

$$
\begin{aligned}
& \mathbf{S 2 2}_{I k+1}^{\prime}=\mathbf{S 2 2}_{I k+1(k)}^{\prime} * \mathbf{A}_{k}^{*}+\mathbf{S 2 2}_{I k+1(k+1)}^{\prime} \mathbf{A}_{k+1}, \\
& \mathbf{S 2 1}_{I k+1}^{\prime}=\mathbf{S 2 1}_{I k+1(k)}^{\prime} * \mathbf{A}_{k}^{*}+\mathbf{S 2 1}_{I k+1(k+1)}^{\prime} \mathbf{A}_{k+1}, \\
& \mathbf{S 2 3}_{I k+1}^{\prime}=\mathbf{S 2 3}_{I k+1(k)}^{\prime} * \mathbf{A}_{k}^{*}+\mathbf{S 2 3}_{I k+1(k+1)}^{\prime} \mathbf{A}_{k+1} .
\end{aligned}
$$

Inside the solid at interfaces $I k$ and $I k+1$ three displacement components can be written as

$$
\underline{\underline{\mathbf{u}}} \mathbf{i}_{I k}=\mathbf{D S} \mathbf{i}_{(I k) k} * \mathbf{A}_{k}^{*}+\mathbf{D S} \mathbf{i}_{(I k) k+1} \mathbf{A}_{k+1},
$$




$$
\underline{\mathbf{u i}}_{I k+1}=\mathbf{D S} \mathbf{i}_{(I k+1) k} * \mathbf{A}_{k}^{*}+\mathbf{D S} \mathbf{i}_{(I k+1) k+1} \mathbf{A}_{k+1},
$$

where $\mathbf{i}$ takes values 1,2 , and 3 to represent three displacement components along $x_{1}, x_{2}$, and $x_{3}$ directions, respectively. The previous matrix expressions are valid for all $n$ +1 interfaces. Therefore, $k$ can take values from 1 to $n+1$.

\section{B. Boundary and continuity conditions}

Across a fluid-solid interface the displacement component normal to the interface should be continuous. Also, at the interface, the transformed negative normal stress $\left(-\mathbf{s 2 2}^{\prime}\right)$ in the solid and the pressure in the fluid should be continuous and the shear stresses must vanish. On the other hand, across a solid-solid interface three displacement components, the normal stress and two shear stresses parallel to the interface must be continuous.

Let the normal velocities at the transducer faces be $\mathbf{V}_{S 0}$ and $\mathbf{V}_{R 0}$, for the lower and upper transducers, respectively. The boundary conditions at the transducer faces are

$$
\begin{aligned}
& \mathbf{M}_{S S} \mathbf{A}_{S}+\mathbf{M}_{S 1} \mathbf{A}_{1}=\mathbf{V}_{S 0} \\
& \mathbf{M}_{R(n+1)} * \mathbf{A}_{n+1}^{*}+\mathbf{M}_{R R} \mathbf{A}_{R}=\mathbf{V}_{R 0} .
\end{aligned}
$$

At the fluid-solid interfaces, the continuity conditions can be expressed similar to what Banerjee and Kundu ${ }^{32}$ presented. At the $k$ th solid-solid interface the continuity conditions are given by

$$
\mathbf{S 2 2}_{k(k-1)}^{\prime} \mathbf{A}_{k-1}^{*}+\mathbf{S 2 2}_{k k}^{\prime} \mathbf{A}_{k}=\mathbf{S 2 2}_{k k^{*}}^{\prime} \mathbf{A}_{k}^{*}+\mathbf{S 2 2}_{k(k+1)}^{\prime} \mathbf{A}_{k+1},
$$

$$
\mathbf{S 2 1}_{k(k-1)}^{\prime}{ }^{*} \mathbf{A}_{k-1}^{*}+\mathbf{S 2 1}_{k k}^{\prime} \mathbf{A}_{k}=\mathbf{S} 2 \mathbf{1}_{k k}^{\prime} * \mathbf{A}_{k}^{*}+\mathbf{S} 2 \mathbf{1}_{k(k+1)}^{\prime} \mathbf{A}_{k+1},
$$

$$
\mathbf{S} 23_{k(k-1)}^{\prime}{ }^{*} \mathbf{A}_{k-1}^{*}+\mathbf{S} 23_{k k}^{\prime} \mathbf{A}_{k}=\mathbf{S} 23_{k k}^{\prime} * \mathbf{A}_{k}^{*}+\mathbf{S} 23_{k(k+1)}^{\prime} \mathbf{A}_{k+1} .
$$

Continuity of three displacement components at the $k$ th interface gives

$$
\mathbf{D S i}_{k(k-1)} \mathbf{A}_{k-1}^{*}+\mathbf{D S i}_{k k} \mathbf{A}_{k}=\mathbf{D S i} \mathbf{i}_{k k} \mathbf{A}_{k}^{*}+\mathbf{D S i} \mathbf{i}_{k(k+1)} \mathbf{A}_{k+1} .
$$

The boundary and continuity conditions can be written in matrix form ${ }^{38}$ :

$$
[\mathbf{M}]\{\mathbf{\Psi}\}=\{\mathbf{V}\} .
$$

The vectors $\{\boldsymbol{\Psi}\}$ and $\{\mathbf{V}\}$ are called source strength vector and force vector, respectively, which can be written as

$$
\begin{aligned}
& \{\boldsymbol{\Psi}\}^{\text {Transpose }}
\end{aligned}
$$

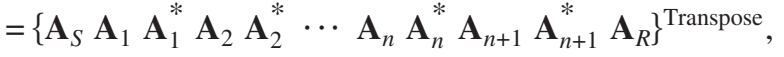

The matrix $\mathbf{M}$ is given by

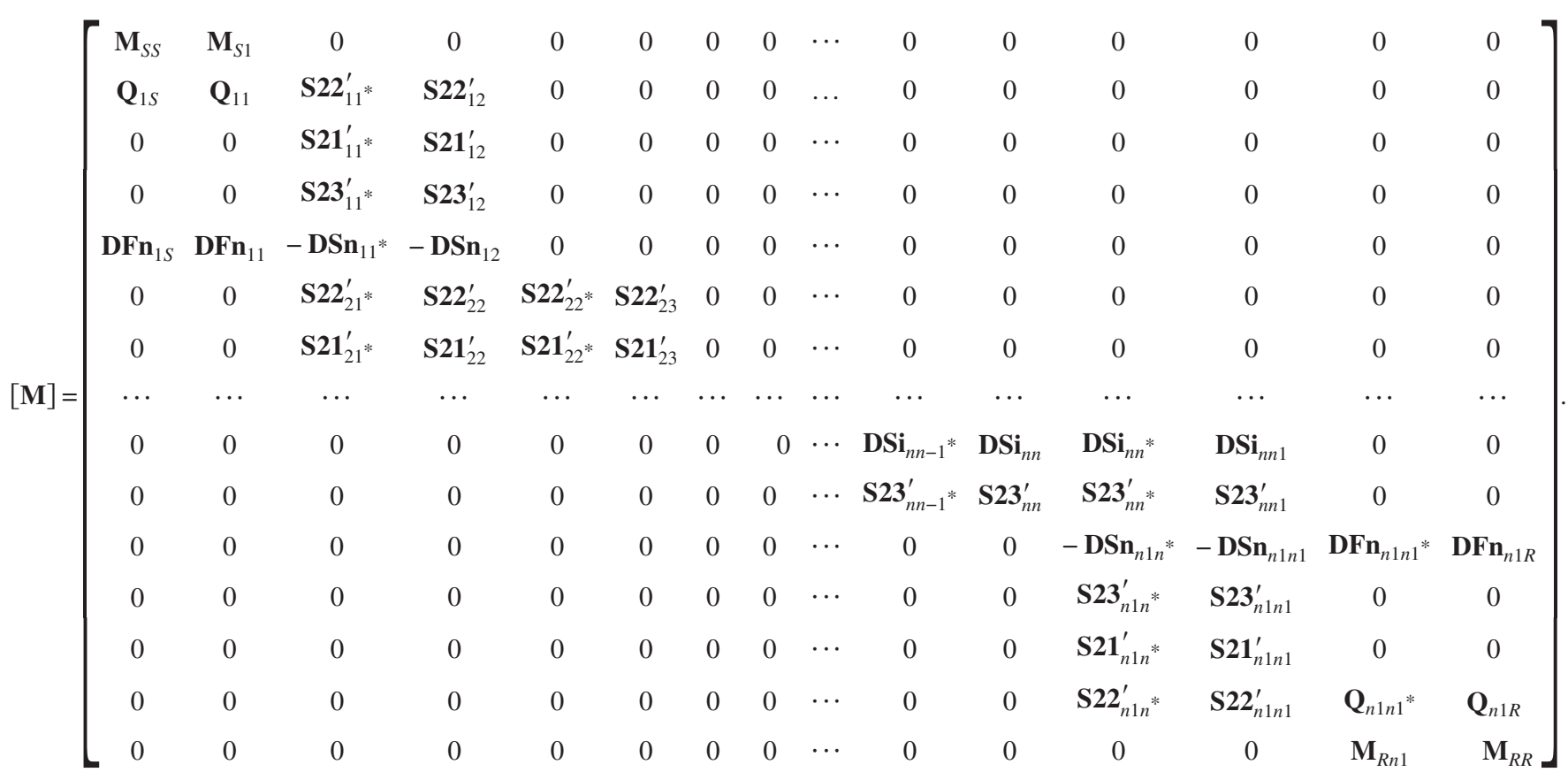


The vector of source strengths $\{\boldsymbol{\Psi}\}$ is obtained by inverting Eq. (28). After calculating the source strengths, the pressure, velocity, stress, and displacement at any point can be obtained by placing the target points there and using corresponding source strength vectors as described earlier by Banerjee and Kundu. ${ }^{38}$ Thus, DPSM can generate threedimensional elastic wave field in a multilayered solid structure.

\section{GREEN'S FUNCTION}

\section{A. Calculation of displacement and pressure Green's functions in fluid}

Spherical bulk wave in a fluid can be generated by a point source in an infinite fluid medium. ${ }^{23}$ If the point source is harmonic, then it will generate harmonic spherical waves. If a point source is generating bulk waves in a fluid, then the harmonic dirac-delta impulsive force will be the body force. Frequency domain pressure Green's function at a point in an infinite fluid medium at a distance of $r$ from the point of excitation is given in Banerjee and Kundu. ${ }^{38}$

\section{B. Calculation of displacement and stress Green's functions in solid}

In DPSM formulation the explicit expressions of both displacement and stress Green's functions are needed. Let the frequency domain displacement Green's function $G_{i j}(\mathbf{x} ; \mathbf{y}, \omega)$ denote the displacement in the $i$ th direction at $\mathbf{y}$ for a point force acting in the $j$ th direction at position $\mathbf{x}$. Therefore, the frequency domain stress Green's tensor for that material can be expressed as $\sigma_{i j}^{q}(\mathbf{x} ; \mathbf{y}, \omega)=\frac{1}{2} C_{i j k l}\left(G_{k q, l}\right.$ $\left.+G_{l q, k}\right)$, when the source is acting in the $q$ th direction placed at $\mathbf{x}$.

The equation of motion for a solid material can be written as

$$
C_{i j k l}\left(x_{n}\right) \frac{\partial^{2}}{\partial x_{j} \partial x_{l}} u_{k}\left(x_{n}, t\right)+F_{i}\left(x_{n}, t\right)=\rho\left(x_{n}\right) \frac{\partial^{2} u_{i}\left(x_{n}, t\right)}{\partial^{2} t},
$$

where $C_{i j k l}\left(x_{n}\right)$ are the material constants, $u_{i}$ are displacement components, $F_{i}$ denotes the body force per unit volume and $i, j, k, l$, and $n$ take values 1,2 , and 3 . In the subsequent formulation all subscripts correspond to usual index notation in three-dimensional space, and $\delta_{i j}$ is the Kronecker delta symbol. In calculations of the Green's functions, inhomogeneity of the material can be incorporated as done by Manolis et al. ${ }^{43}$ Let us consider here only homogeneous materials and $C_{i j k l}=C_{j i k l}=C_{k l i j}=C_{i k j l}$ to satisfy the symmetry condition. The external force in Eq. (32) can be considered as an impulsive force at origin with magnitude $\mathbf{P}$ :

$$
\mathbf{F}(\mathbf{x}, t)=\mathbf{P} f(t) \delta(\mathbf{x}) \quad \text { or } \quad F_{i}=P_{i} f(t) \delta\left(x_{j}\right)
$$

where $\mathbf{P}$ denotes the source strength. From Eqs. (32) and (33) one can write

$$
C_{i j k l} \frac{\partial^{2}}{\partial x_{j} \partial x_{l}} u_{k}\left(x_{n}, t\right)+P_{i} f(t) \delta\left(x_{n}\right)=\rho \frac{\partial^{2} u_{i}\left(x_{n}, t\right)}{\partial^{2} t} .
$$

The Green's function for different materials can be solved in different ways from the above equation. Expressions of displacement and stress Green's functions are given for isotropic and anisotropic materials as

$$
\begin{aligned}
G_{i j}(\mathbf{x} ; \mathbf{y}, \omega)= & \frac{1}{4 \pi \rho \omega^{2}}\left[\frac { e ^ { i k _ { p } r } } { r } \left(k_{p}^{2} R_{i} R_{j}+\left(3 R_{i} R_{j}-\delta_{i j}\right)\left(\frac{i k_{p}}{r}\right.\right.\right. \\
& \left.\left.-\frac{1}{r^{2}}\right)\right)+\frac{e^{i k_{s} r}}{r}\left(k_{s}^{2}\left(\delta_{i j}-R_{i} R_{j}\right)-\left(3 R_{i} R_{j}\right.\right. \\
& \left.\left.\left.-\delta_{i j}\right)\left(\frac{i k_{s}}{r}-\frac{1}{r^{2}}\right)\right)\right], \quad R_{i}=\frac{x_{i}-y_{i}}{r} \\
& r=|\mathbf{x}-\mathbf{y}| .
\end{aligned}
$$

$G_{i j}(\mathbf{x} ; \mathbf{y}, \omega)$ is the displacement Green's function for isotropic materials.

Similarly considering a point force at the origin the displacement Green's function in anisotropic materials can be written as ${ }^{42}$

$$
\begin{aligned}
G_{i j}(\mathbf{x} ; \mathbf{0}, \omega)= & \frac{i}{8 \pi^{2}} \int_{|\mathbf{n}|=1} \sum_{M=1}^{3} \frac{k_{M} g_{i}^{M} g_{j}^{M}}{2 \rho c_{M}^{2}} \\
& \times \exp \left(i\left(k_{M} n_{p} x_{p}\right)\right) d \Omega\left(n_{m}\right) \\
& +\frac{1}{8 \pi^{2}} \int_{|\mathbf{n}|=1} \sum_{M=1}^{3} \frac{g_{i}^{M} g_{j}^{M}}{\rho c_{M}^{2}} \delta\left(n_{p} x_{p}\right) d \Omega\left(n_{m}\right)
\end{aligned}
$$

The stress Green's function in isotropic solids ${ }^{38}$

$$
\sigma_{i j}^{q}(\mathbf{x} ; \mathbf{y}, \omega)=\left(\mu\left(G_{i k, j}+G_{k j, i}\right) \delta_{k q}+\lambda \delta_{i j} G_{k q, k}\right)
$$

and the stress Green's function in anisotropic materials ${ }^{42}$ can be obtained from the literature,

$$
\begin{aligned}
\sigma_{i j}^{q}(\mathbf{x} ; \mathbf{0}, \omega)= & \int_{-\infty}^{+\infty}\left[\frac{C_{i j k l} H(t)}{8 \pi^{2}} \sum_{M=1}^{3} \int_{|\mathbf{n}|=1} \frac{n_{l} g_{k}^{M} g_{q}^{M}}{\rho c_{M}^{3}} \frac{\partial^{2}}{\partial t^{2}}\right. \\
& \left.\times\left[\delta\left(c_{M} t-n_{p} x_{p}\right)\right] d \Omega\left(n_{m}\right)\right] e^{i \omega t} d t
\end{aligned}
$$

For the previous equation the point force is acting along the $q$ direction.

\section{NUMERICAL RESULTS}

Accuracy of the DPSM modeling for the wave field computation in a homogeneous solid with planar and nonplanar boundaries has been shown by Banerjee and Kundu. ${ }^{35}$ In this article the results are presented for an inhomogeneous multilayered plate composed of two different isotropic layers. Both the plate and the ultrasonic transducers are immersed in water that acts as the coupling fluid for the ultrasonic signals between the transducers and the plate, see Fig. 1. Results are presented for both planar and nonplanar interfaces. Continuity conditions on stresses and displacements across the interface are checked to indirectly verify the accuracy of the computed results. Two transducers, one above and one below the plate are placed at the horizontal position $x_{1}=0$. The distance between a transducer face (at the center 


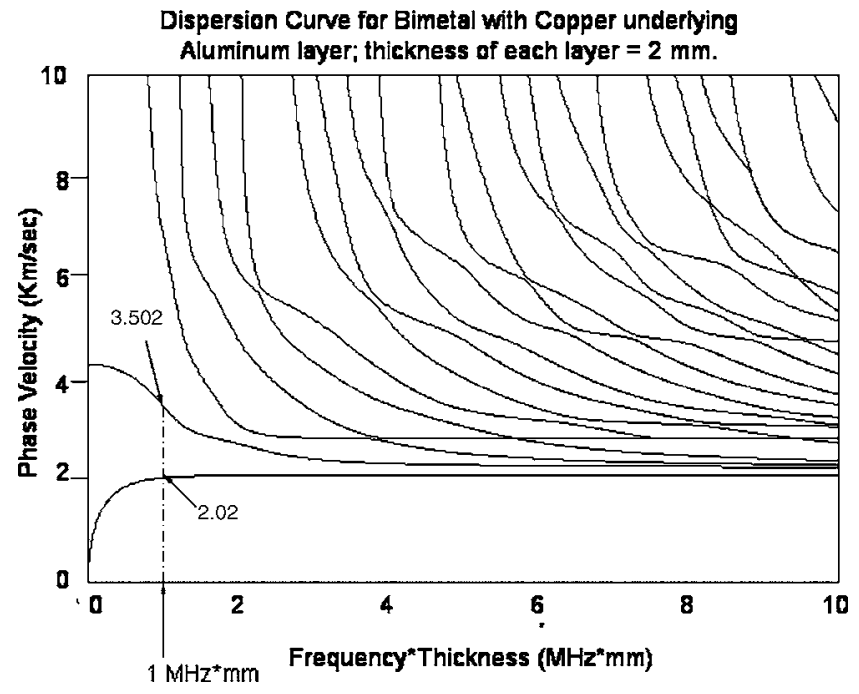

FIG. 2. Dispersion curves for the $4 \mathrm{~mm}$ thick bimetal (aluminum-copper) plate.

point) and its nearest fluid-solid interface is $6 \mathrm{~mm}$. The total plate thickness is $4 \mathrm{~mm}$. Therefore, the face to face distance between the two transducers along $x_{2}$ axis is $16 \mathrm{~mm}$. The transducers have $4 \mathrm{~mm}$ diameter. The plate length and width in the in-plane and the out-of-plane directions, respectively, are much greater than the transducer diameter. The ultrasonic field is computed along a plane which bisects the transducers and the plate and thus forms a plane of symmetry of the problem geometry. Note that we are solving a threedimensional problem and plotting the field along the vertical plane of symmetry of the problem geometry. One hundred point sources are distributed near each transducer face and additional point sources are placed along the plate boundaries and interfaces, as shown in Fig. 1. The average distance between two neighboring point sources is approximately $0.4 \mathrm{~mm}$. Now the question is how many point sources should be used to model the interfaces and plate boundaries that are extended to infinity in both in-plane and out-of-plane directions. Point sources in the vertical plane of symmetry consisting of several lines of point sources as shown in Fig. 1 are first distributed and the ultrasonic field is computed along the central plane. Then two more planes of point sources are added on the two sides of the central plane and the field is computed again at the central plane. This process of adding two planes of point sources on two sides of the central plane is continued until the computed field at the central plane is converged. Note that the additional planes of point sources on two sides of the central plane are applied only along the plate boundaries and interfaces whose dimensions in the outof-plane directions are large. For the finite dimension transducers 100 point sources are placed over the total transducer face from the very beginning. Interestingly, the results are found to converge with only three planes of point sources in the out-of-plane direction. However, if one is interested in computing the ultrasonic field at another plane which is not necessarily the plane of symmetry then more planes of point sources might be necessary.

On each side of an interface or a plate boundary 35 point sources are distributed on the central plane. Sources are
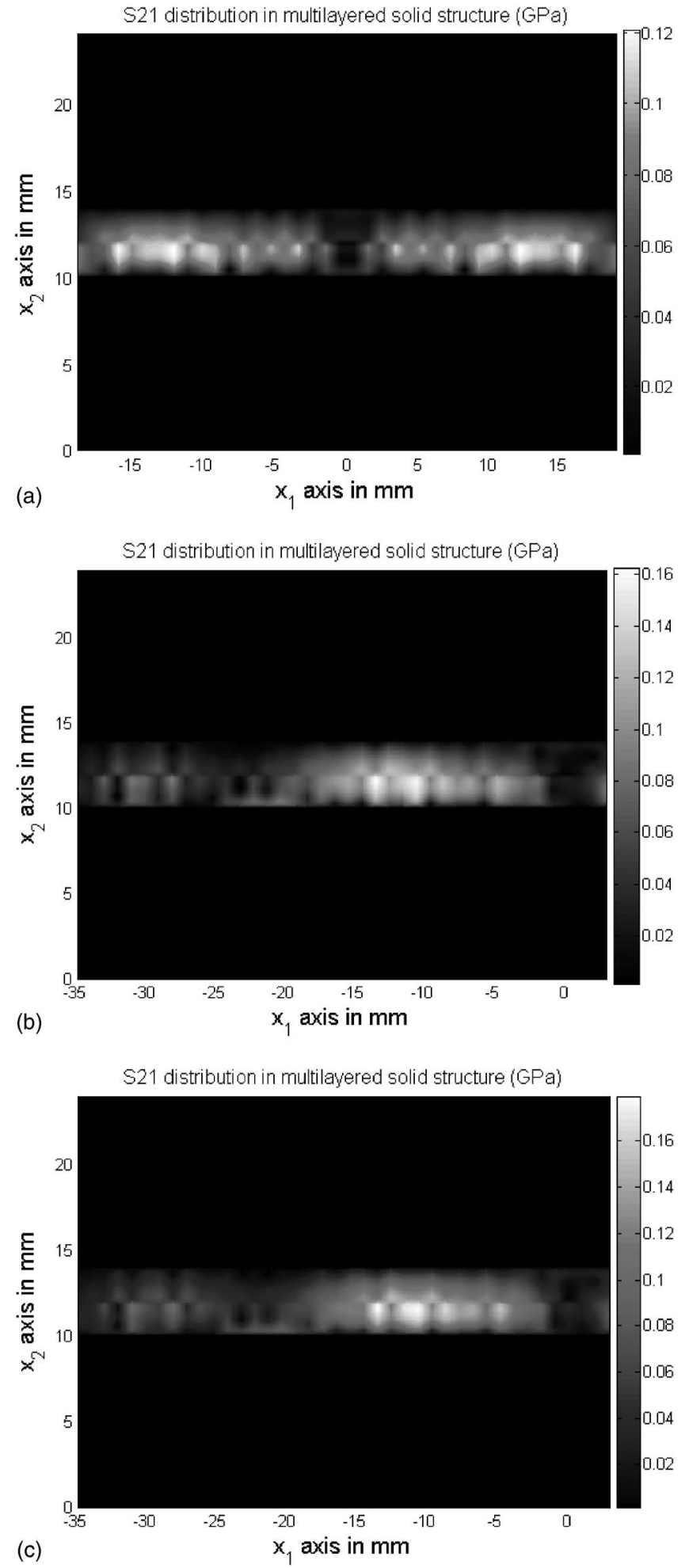

FIG. 3. Shear stress variations in the $4 \mathrm{~mm}$ thick bimetal (aluminumcopper) plate generated by two ultrasonic transducers placed on $x_{1}=0$ line. One transducer is positioned below $\left(x_{2}=0\right)$ and the second one is above $\left(x_{2}=24 \mathrm{~mm}\right)$ the plate. Results for normal incidence (top, $\left.\theta=0^{\circ}\right)$, and inclined incidences (middle, $\theta=25^{\circ}$ and bottom, $\theta=47.1^{\circ}$ ), are shown.

placed along the interfaces and plate boundaries in the illuminated region as well as well beyond this region. A total of 105 sources are then necessary to model the interface with three planes of point sources in the out-of-plane direction. Increasing the number of sources to 175 to construct five 
planes of point sources in the out-of-plane direction did not change the computed ultrasonic field in the central plane. The number of point sources taken for the wave field computation is based on the convergence criterion of the DPSM technique. ${ }^{18}$ The convergence of the problem solution has been also tested by increasing the number of point sources in one plane of point sources and at the transducer face. When the spacing between two neighboring point sources is less than one-third wavelength then the problem is found to converge. Further increase in the number of point sources did not change the computed results significantly. For most of the results presented in this article the distance between two neighboring point sources has been kept at wavelength $/ \pi$. Note that 35 point sources in each layer of an interface are distributed over $40 \mathrm{~mm}$ length along $x_{1}$ axis. Hence, distance between two neighboring point sources is approximately $1.14 \mathrm{~mm}$ (less than the shortest wavelength $/ \pi$ ), thus the results presented here are well converged. The boundary and continuity conditions are enforced at the apex of the spherical bulbs [little spheres shown in Fig. 1(a)] of the point sources. Apex point is the point where a spherical bulb of a point source touches the problem boundary or interface. Therefore, the number of points where boundary and continuity conditions are enforced is the same as the number of point sources. For example, along the $x_{1}$ axis along every line of point sources there are 35 points on the boundary of the plate to enforce continuity conditions. On the transducer surface there are 100 points to enforce boundary conditions. The number of boundary and continuity conditions is equal to the number of unknowns (point source strengths). Wave fields in the layered solid plate are generated for different striking angles of the ultrasonic beams. Results presented in the following show displacement and stress variations along the length and depth of the plate specimen.

A two layered plate composed of aluminum and copper is considered for the analysis. The properties of aluminum are $c_{p}=6.2 \mathrm{~km} / \mathrm{s}, c_{s}=3.04 \mathrm{~km} / \mathrm{s}$, and $\rho=2.7 \mathrm{gm} / \mathrm{cm}^{3}$, where $c_{p}$ is the $P$-wave speed, $c_{s}$ is the $S$-wave speed, and $\rho$ is the density. The properties of copper are $c_{p}=4.6 \mathrm{~km} / \mathrm{s}, c_{s}$ $=2.26 \mathrm{~km} / \mathrm{s}$, and $\rho=8.9 \mathrm{gm} / \mathrm{cm}^{3}$. It is assumed that the aluminum layer of thickness $t=2 \mathrm{~mm}$ underlines the copper layer of thickness $t=2 \mathrm{~mm}$. Wave fields are generated with $250 \mathrm{kHz}$ transducers. It is well known that in a multilayered plate the wave modes propagate at certain frequencies with certain phase velocities. Wave modes obtained from the dispersion equation are in general dispersive; dispersion curves for this two-layered plate are shown in Fig. 2.

From the dispersion curves one can see that at $250 \mathrm{kHz}$ frequency there are two guided wave modes. For a given frequency for generating a specific guided wave mode one can obtain the critical angle of incidence of the striking ultrasonic beam from the phase velocities (shown along the vertical axis of the dispersion curve plot) using Snell's law,

$$
\theta_{\mathrm{cr}}=\sin ^{-1}\left(\frac{c_{f}}{c_{\mathrm{ph}}}\right)
$$

where $c_{f}$ is the acoustic wave speed in the coupling fluid, $\theta_{\mathrm{cr}}$ is the critical angle of incidence for generating the guided wave mode whose phase velocity is $c_{\mathrm{ph}}$.
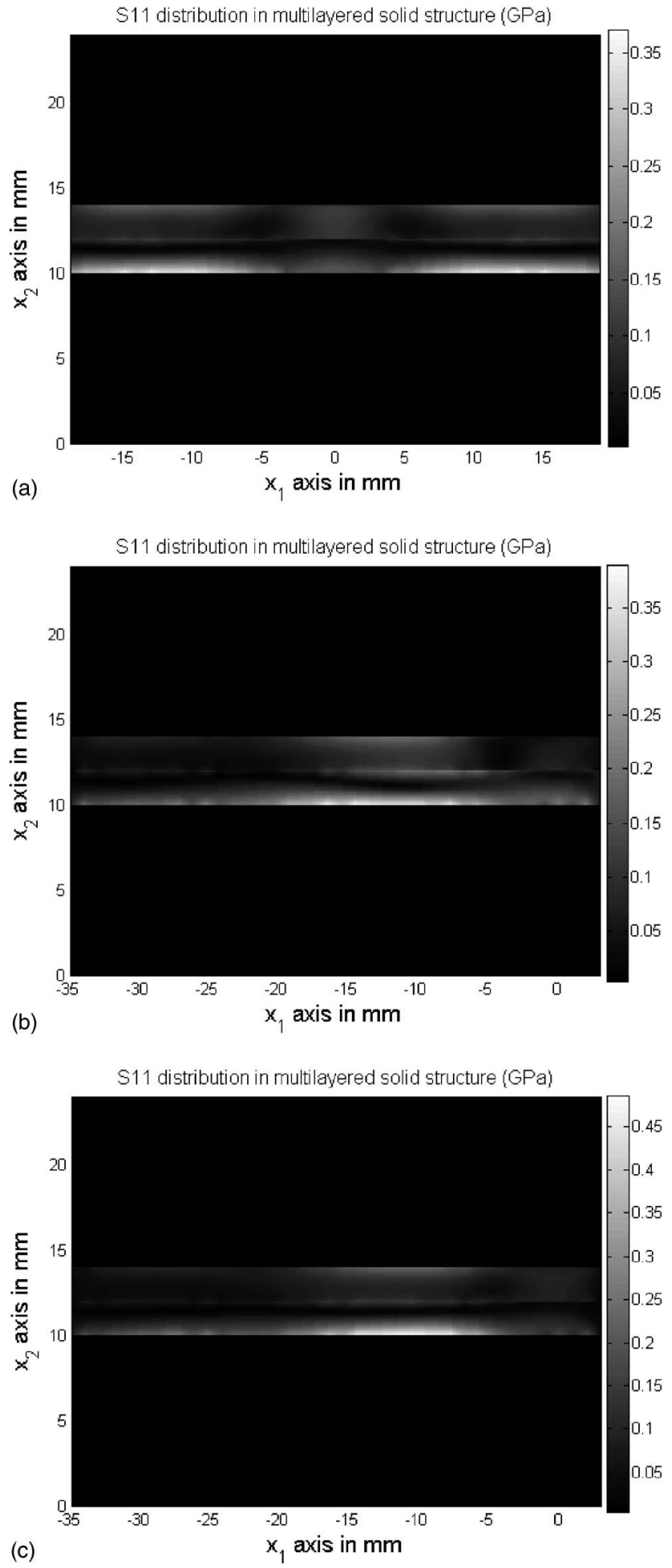

FIG. 4. For the plate and transducer orientations shown in Fig. 1(a) and described in Fig. 3, variations of $\sigma_{11}$ are shown in the left and right columns, respectively. Three rows show results for normal incidence (top row, $\theta=0^{\circ}$ ), and inclined incidences (middle row $\theta=25^{\circ}$ and bottom row $\theta=47.1^{\circ}$ ).

Thus, for $250 \mathrm{kHz}$ signal frequency one obtains critical angles $47.1^{\circ}$ and $25^{\circ}$ for generating Modes 1 and 2, respectively, in the plate specimen. Stress and displacement variations in the plate for the normal incidence $\left(\theta=0^{\circ}\right)$ as well as for the two critical angles of incidence $\left(\theta=25^{\circ}\right.$ and $\left.47.1^{\circ}\right)$ are shown in Figs. 3-5. The shear stress $\sigma_{21}$ (or $S_{21}$ ) variations in the plate for these three angles of incidence are shown in Fig. 


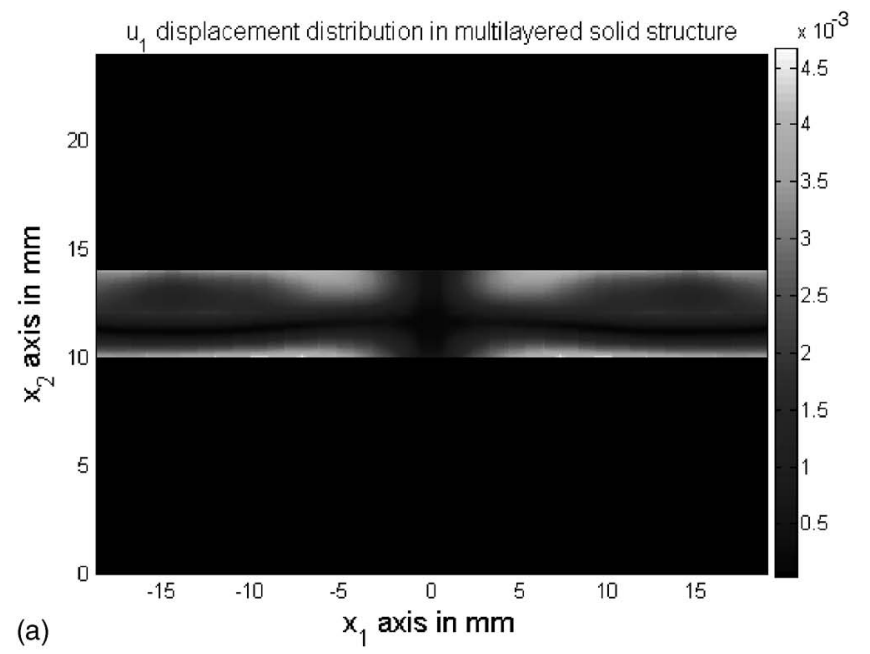

(a)

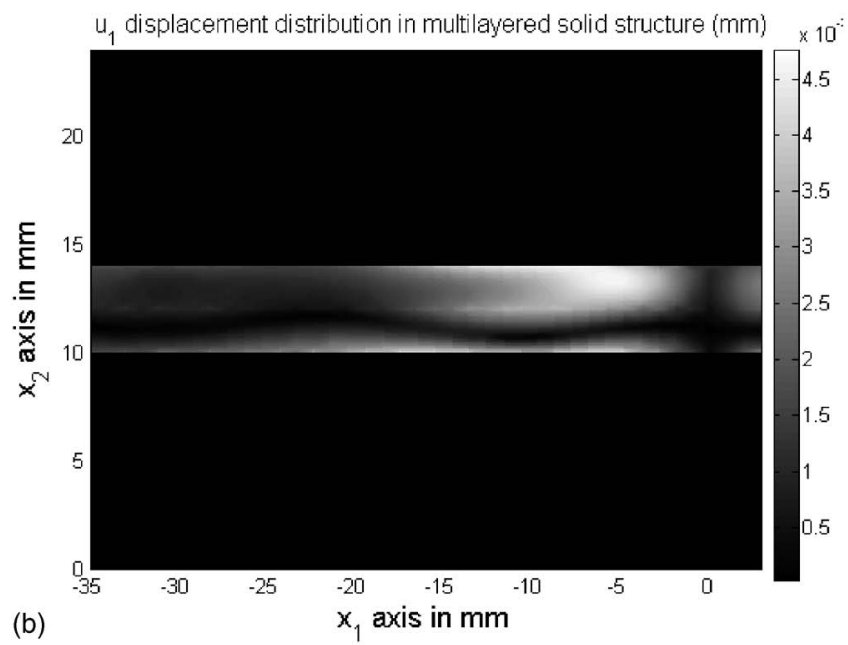

(b)

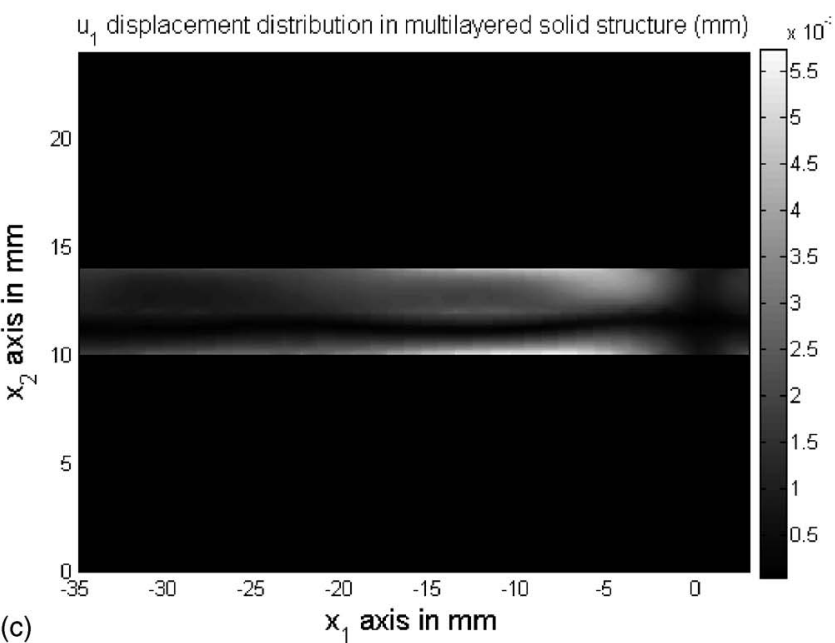

FIG. 5. Horizontal displacements inside the plate for the plate and transducer orientations shown in Fig. 1(a) and described in Fig. 3. Three rows show results for normal incidence (top row, $\theta=0^{\circ}$ ), and inclined incidences (middle row $\theta=25^{\circ}$ and bottom row $\theta=47.1^{\circ}$ ).

3 , variations of the normal stress component $\sigma_{11}$ (or $S_{11}$ ) are shown in Fig. 4 and that for the displacement component $\left(u_{1}\right)$ are shown in Fig. 5. As expected, for the normal incidence all displacement and stress amplitudes are symmetric about the $x_{2}$ axis $\left(x_{1}=0\right.$ line $)$ since the ultrasonic beam strikes the plate at $x_{1}=0$. Such symmetry is not observed for

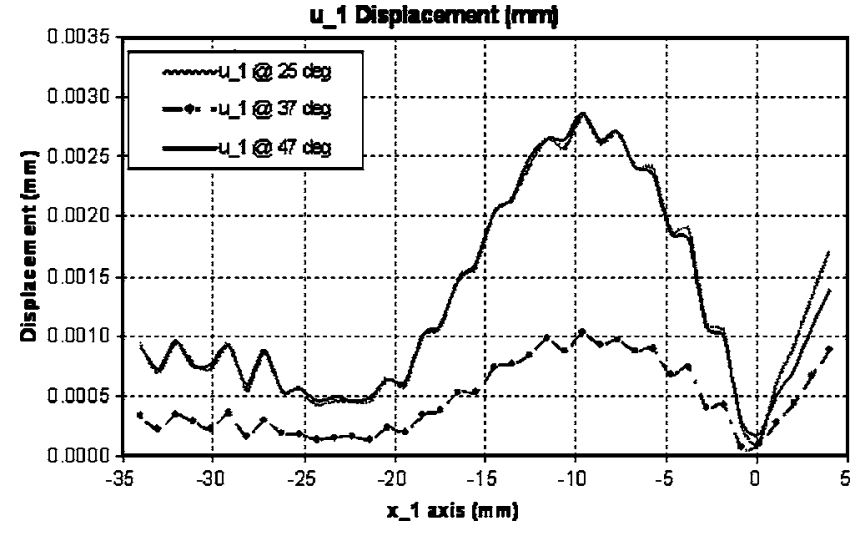

FIG. 6. Horizontal displacement $\left(u_{1}\right)$ variation along the interface of the bimetal plate for three different transducer inclination angles.

the oblique incidence. Figure 6 shows the horizontal displacement component $\left(u_{1}\right)$ along the interface of the plate for three different transducer inclination angles. It can be seen from this figure that when the transducers are inclined at critical angles $\left(47^{\circ}\right.$ and $\left.25^{\circ}\right)$ the displacement values at the interface away from the striking beam are significantly higher than that for the noncritical angle $\left(\theta=37^{\circ}\right)$ of incidence. The higher displacement values for the critical angle of incidence are expected because of the propagating wave modes. Similar phenomenon is observed for the $u_{2}$ displacement component as well.

From the gray scale images of Figs. $3-5$ it is not clear if the continuity conditions (continuity of $u_{1}, u_{2}, \sigma_{22}$, and $\sigma_{12}$ ) across the interface and the boundary condition $\left(\sigma_{12}=0\right)$ at the top and bottom boundaries are satisfied. These conditions can be verified from the line plot in Figs. 7. Shear stress variation along the plate thickness from the bottom boundary $\left(x_{2}=10 \mathrm{~mm}\right)$ to the top boundary $\left(x_{2}=14 \mathrm{~mm}\right)$ at $x_{1}=$ $-15 \mathrm{~mm}$ is shown in Fig. 7 for the normal incidence of the ultrasonic beam. Three curves in this figure correspond to real, imaginary and absolute values, respectively. In Fig. 7

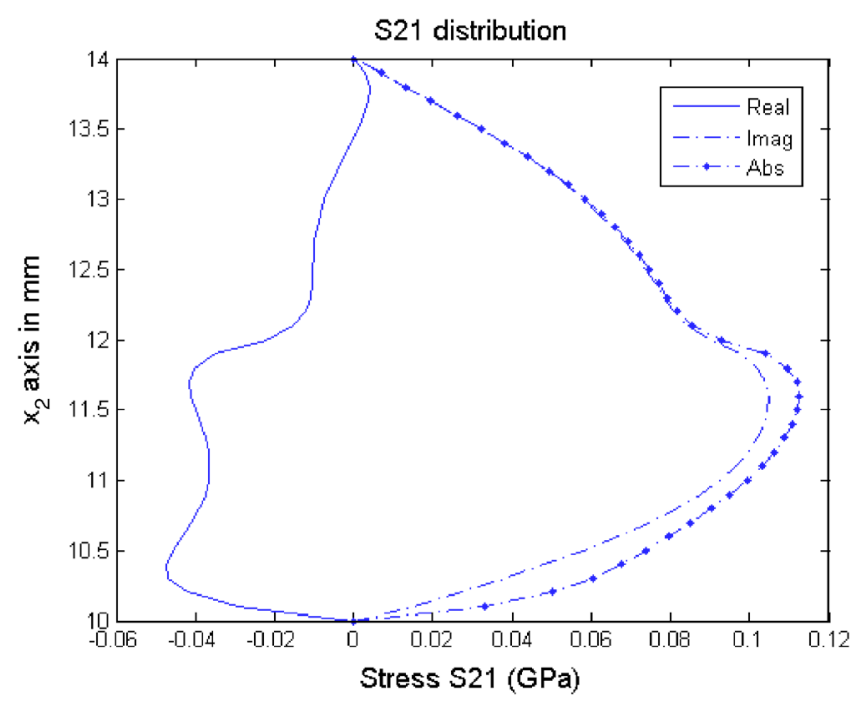

FIG. 7. (Color online) Shear stress variation across the $4 \mathrm{~mm}$ thick bimetal plate at $x_{1}=-15 \mathrm{~mm}$ for normal incidence. Note that it is continuous across the interface at $x_{2}=12 \mathrm{~mm}$ and zero at the top $\left(x_{2}=14 \mathrm{~mm}\right)$ and bottom $\left(x_{2}=10 \mathrm{~mm}\right)$ boundaries. 

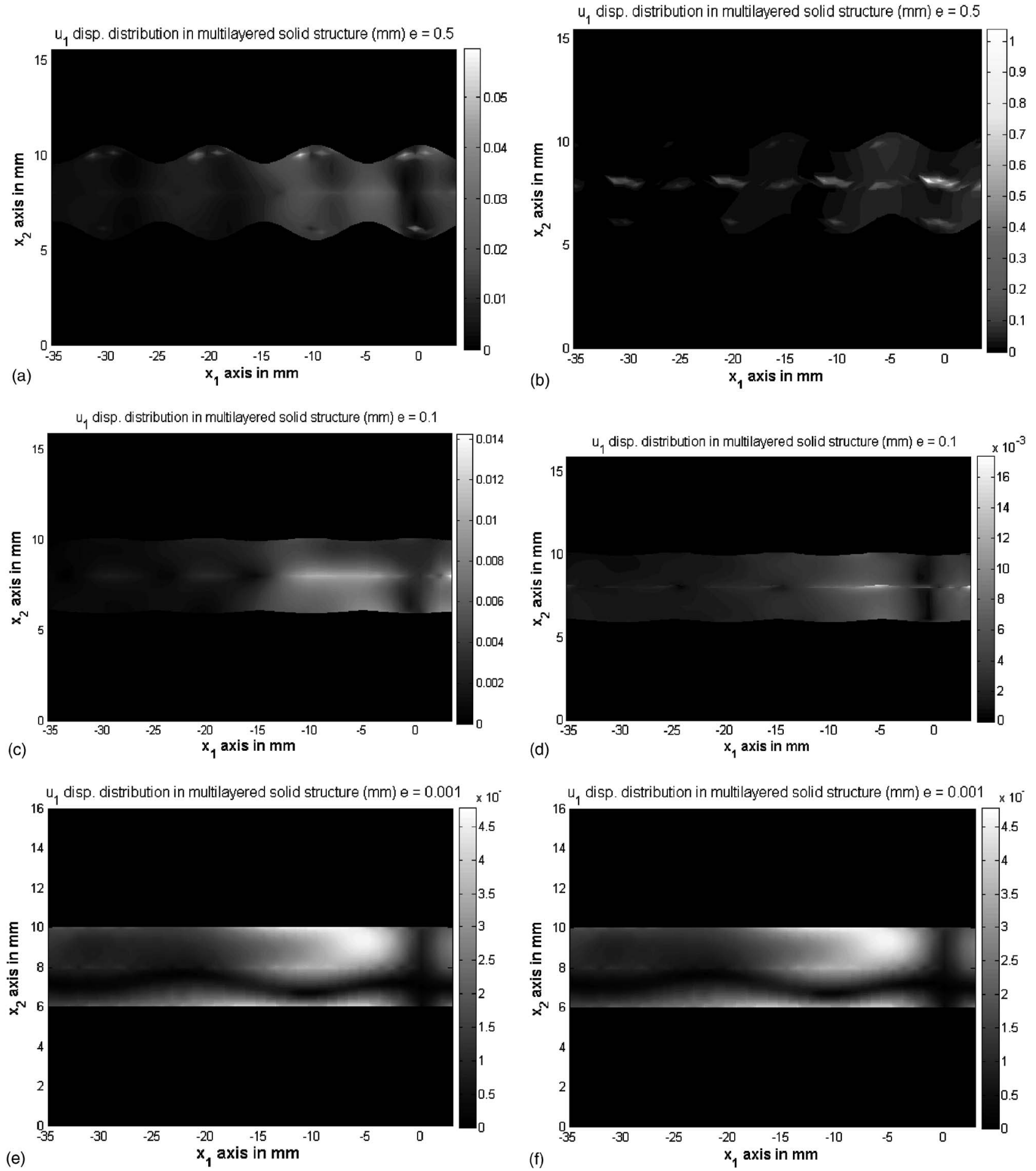

FIG. 8. Horizontal displacements inside the corrugated plate for the plate and transducer orientations shown in Fig. 1 and described in Fig. 3. The left column $(\mathrm{a}, \mathrm{c}, \mathrm{e})$ is for symmetric corrugation and the right column $(\mathrm{b}, \mathrm{d}, \mathrm{f})$ is for antisymmetric corrugation. Three rows show results for three different amplitudes of corrugation. Corrugation amplitude (e) is $0.5 \mathrm{~mm}$ for the top row (a), $0.1 \mathrm{~mm}$ for the middle row (b), and 0.001 for the bottom row (c). Incident angle of the ultrasonic beam is $25^{\circ}$.

one can clearly see that the shear stress is zero at the top and bottom boundaries and continuous across the interface $\left(x_{2}\right.$ $=12 \mathrm{~mm}$ ). Similar plots (not shown) shows discontinuities in $\sigma_{11}$ at the interface but no such jump at the interface is observed in $\sigma_{22}$ plot. The displacement components $u_{1}$ and $u_{2}$ show continuity across the interface and nonzero values at the plate boundaries.
Finally, the effect of the non-planar boundary surfaces and interface of the layered plate is investigated and presented in Figs. 8 and 9. The top and bottom surfaces of the plate are made nonplanar by replacing the flat plate boundaries by sinusoidal boundaries. For the symmetrically corrugated plate the central interface remains flat but for the antisymmetric corrugation the solid-solid interface is also made 

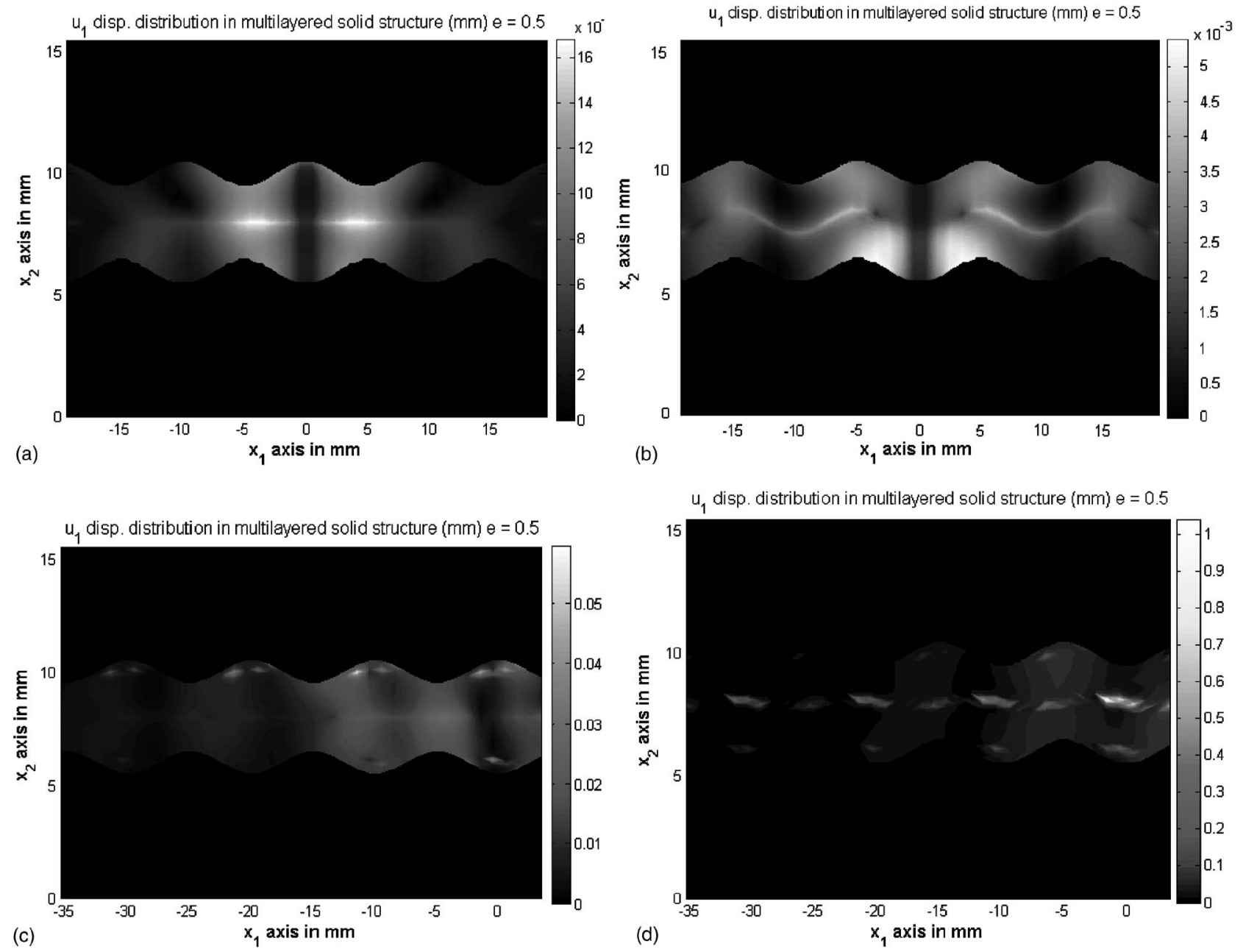

FIG. 9. Horizontal displacements inside the corrugated plate (corrugation amplitude is $0.5 \mathrm{~mm}$ ) for the plate and transducer orientations shown in Fig. 1 and described in Fig. 3. The left column (a,c,e) is for a symmetric corrugation and the right column (b,d,f) is for antisymmetric corrugation. Two rows show results for normal incidence (top row, $\theta=0^{\circ}$ ), and inclined incidences (bottom row $\theta=25^{\circ}$.

corrugated, as shown in left (a, c, e) and right images (b, d, f) of Figs. 8(a) and 9. The wavelength of the corrugation is $10 \mathrm{~mm}$. The corrugation amplitude $(e)$ is $0.5 \mathrm{~mm}$ for the top images [Figs. 8(a) and 8(b)], $0.1 \mathrm{~mm}$ for the middle images [Figs. 8(c) and $8(\mathrm{~d})]$ and $0.001 \mathrm{~mm}$ for the bottom images [Figs. 8(e) and 8(f)]. Note that the height of the peak and the depth of the dip are both equal to the corrugation amplitude $e$. Therefore, the vertical distance between the peak and the dip is $2 e$. Figure 8 shows the horizontal displacement $\left(u_{1}\right)$ inside the two corrugated plates for $25^{\circ}$ angle of strike. Transducer locations are the same as those for the flat plate. Note how the ultrasonic fields change with the corrugation amplitude and type of corrugation (symmetric and antisymmetric). For very small corrugation amplitude (e $=0.001 \mathrm{~mm}$ ), as expected, the ultrasonic fields inside the symmetric and antisymmetric corrugated plates match with that for the flat plate. For high corrugation amplitude $(e$ $=0.5)$ the field is significantly different for symmetric and antisymmetric corrugations. Figure 9 shows the ultrasonic field ( $u_{1}$ variation) for two different angles of incidence for the striking ultrasonic beam; these are $0^{\circ}$ and $25^{\circ}$, shown in top and bottom rows, respectively.

\section{CONCLUSIONS}

DPSM technique has been generalized in this article for modeling ultrasonic fields inside multilayered solids. Here finite size ultrasonic transducers generate the ultrasonic fields unlike most studies by the earlier investigators where plane waves of infinite width were assumed to be the incident field. Step by step mathematical derivation of the DPSM formulation for multilayered plates, excited by finite-size ultrasonic transducers, has been presented in the article. Ultrasonic fields in bimaterial plates having planar and nonplanar boundaries and interfaces are given and the satisfaction of the boundary and continuity conditions at the interfaces has been checked. Readers who are interested in solving multilayered problems of different geometry can develop their own formulation and computer codes following the mathematical steps outlined in the article. The main objective of this article was to extend the technique to nonhomogeneous solids. In future publications nonhomogenous solids with internal voids and cracks will be modeled. 


\section{ACKNOWLEDGMENT}

The authors thank Mr. Samik Das, graduate student at the University Arizona, Tucson for generating the dispersion curves for the multilayered solid plate presented in this article. This research was partially supported from NSF Grant Nos. CMS-9901221, CMMI-0226466, CMMI-0443494, CMMI-0530991, and OISE-0352680. The results and conclusions presented here are those of the authors and do not represent the view of NSF

${ }^{1}$ J. W. S. Rayleigh, The Theory of Sound (Dover, New York, 1945), Vol. II. ${ }^{2}$ R. Stoneley, "The seismological implications of aeolotropy in continental structure," Mon. Not. R. Astr. Soc. of London, Geophys. Suppl. 5, 343 349 (1949).

${ }^{3} \mathrm{~W}$. T. Thompson, "Transmission of elastic waves through a stratified solid medium," J. Appl. Phys. 21, 89-93 (1950).

${ }^{4}$ N. A. Haskell, "The dispersion of surface waves on multilayered media," Bull. Seismol. Soc. Am. 43, 17-21 (1953).

${ }^{5}$ T. Kundu and A. K. Mal, "Elastic waves in a multilayered solid due to a dislocation source," Wave Motion 7, 459-471 (1985).

${ }^{6}$ J. L. Rose, Ultrasonic Waves in Solid Media (Cambridge University Press, New York, 1999).

${ }^{7}$ G. R. Liu and Z. C. Xi, Elastic Waves in Anisotropic Laminates (CRC Press, Boca Raton, FL, 2002).

${ }^{8}$ Ultrasonic Nondestructive Evaluation: Engineering and Biological Material Characterization, edited by T. Kundu (CRC Press, Boca Raton, FL, 2004), Chaps. 2 and 4.

${ }^{9}$ B. Biondi, "Solving the frequency-dependent Eikonal equation," 62nd Annual International Meeting, Society of Exploration of Geophysics, Expanded Abstracts (1992), p. 1315.

${ }^{10} \mathrm{~V}$. Cervený and I. Psencik, "Gaussian beams and paraxial ray approximation in three-dimensional elastic inhomogeneous media," J. Geophys. $\mathbf{5 3}$ 1-9 (1983).

${ }^{11}$ PZFlex Software, Version: 1-j.6 (Weilinger Associates, Inc., Washington, DC, 2001).

${ }^{12} \mathrm{X}$. Zhao and J. L. Rose, "Boundary Element Modeling for Defect Characterization potential in a wave guide," Int. J. Solids Struct. 40, 26452658 (2003).

${ }^{13}$ R. P. Shaw, "Boundary integral equation methods applied to wave problems," Developments in Boundary Element Methods - 1, edited by P. K. Banerjee and R. Butterfield (Applied Science Publications, London, 1979), Chap. 6, pp. 121-153.

${ }^{14}$ B. P. Newberry and R. B. Thompson, "A paraxial theory for the propagation of ultrasonic beams in anisotropic solids," J. Acoust. Soc. Am. 85, 2290-2300 (1989).

${ }^{15}$ J. J. Wen and M. A. Breazeale, "A diffraction beam field expressed as the superposition of Gaussian beams," J. Acoust. Soc. Am. 83, 1752-1756 (1988).

${ }^{16}$ M. Spies, "Transducer field modeling in anisotropic media by superposition of Gaussian base functions," J. Acoust. Soc. Am. 105, 633-638 (1999).

${ }^{17}$ L. W. Schmerr, H. J. Kim, R. Huang, and A. Sedov, "Multi-Gaussian ultrasonic beam modeling," Proceedings of the World Congress of Ultrasonics (WCU 2003), Paris, September 7-10 (2003), Vol. 93.

${ }^{18}$ M. Spies, "Analytical methods for modeling of ultrasonic nondestructive testing of anisotropic media," Ultrasonics 42, 213-219 (2004).

${ }^{19} \mathrm{C}$. Rajamohan and J. Raamachandran, "Bending of anisotropic plates by charge simulation method," Adv. Eng. Software 30, 369-376 (1999).

${ }^{20} \mathrm{~B}$. Ch. Hafner, "The multiple multipole method (MMP) in electro and magnetostatic problems," IEEE Trans. Magn. 19, 2367-2370 (1983).

${ }^{21}$ Ch. Hafner, "MMP calculations of guided waves," IEEE Trans. Magn. 21,
2310-2312 (1985).

${ }^{22}$ M. G. Imhof, "Computing the elastic scattering from inclusions using the multiple multipoles method in three dimensions," Geophys. J. Int. 156, 287-290 (2004).

${ }^{23}$ F. J. Sanchez-Sesma and J. A. Esquivel, "Ground motion on alluvial valleys under incident planes SH waves," Bull. Seismol. Soc. Am. 69, 11071111 (1979).

${ }^{24}$ H. L. Wong, "Diffraction of P, SV, and Rayleigh waves by surface topography," Bull. Seismol. Soc. Am. 72, 1167-1171 (1982).

${ }^{25}$ M. Dravinski and T. K. Mossessian, "Scattering of plane harmonic P, SV, and Rayleigh waves by dipping layers of arbitrary shape," Bull. Seismol. Soc. Am. 77, 212-215 (1987).

${ }^{26}$ F. J. Sanchez-Sesma and M. Campillo, "Diffraction of P, SV and Rayleigh waves by topographic features: A boundary integral formulation," Bull. Seismol. Soc. Am. 81, 2234-2253 (1991).

${ }^{27}$ F. J. Sanchez-Sesma and M. Campillo, "Topographic effects for incident P, SV and Rayleigh waves," Tectonophysics 218, 113-125 (1993).

${ }^{28}$ T. Pointer, E. Liu, and J. A. Hudson, "Numerical modeling of seismic waves scattered by hydrofractures: application of the indirect boundary element method," Geophys. J. Int. 135, 289-303 (1998).

${ }^{29}$ M. Bouchon and F. J. Sanchez-Sesma, "Boundary integral equations and boundary elements methods in elastodynamics," in Advances in Wave Propagation in Heterogeneous Earth, Advances in Geophysicis, Vol. 48, edited by R.-S. Wu, V. Maupin, and R. Dmowska (Elsevier-Academic, New York, 2007).

${ }^{30}$ D. Placko and T. Kundu, "A theoretical study of magnetic and ultrasonic sensors: Dependence of magnetic potential and acoustic pressure on the sensor geometry," Proc. SPIE, 4335, 52-62 (2001).

${ }^{31}$ S. Banerjee, T. Kundu, and D. Placko, "Ultrasonic field modelling in multilayered fluid structures using DPSM technique," ASME J. Appl. Mech. 73, 598-609 (2006)

${ }^{32} \mathrm{~S}$. Banerjee and T. Kundu, "DPSM technique for ultrasonic field modelling near fluid-solid interface," Ultrasonics 46, 235-250 (2007).

${ }^{33} \mathrm{~S}$. Banerjee and T. Kundu, "Semi-analytical modeling of ultrasonic fields in solids with internal anomalies immersed in a fluid," Wave Motion (in press).

${ }^{34} \mathrm{~S}$. Banerjee and T. Kundu, "Ultrasonic field modeling in plates immersed in fluids," Int. J. Solids Struct. 44, 6013-6029 (2007).

${ }^{35} \mathrm{~S}$. Banerjee and T. Kundu, "Elastic wave propagation in sinusoidally corrugated wave guides," J. Acoust. Soc. Am. 119, 2006-2017 (2006).

${ }^{36}$ T. Kundu, J.-P. Lee, C. Blase, and J. Bereiter-Hahn, "Acoustic microscope lens modeling and its application in determining biological cell properties from single and multi-layered cell models," J. Acoust. Soc. Am. 120, 1646-1654 (2006).

${ }^{37}$ A. K. Mal and S. J. Singh, Deformation of Elastic Solids (Prentice Hall, Englewood Cliffs, NJ, 1991).

${ }^{38}$ S. Banerjee and T. Kundu, "Chapter 4: Advanced Applications of Distributed Point Source Method-Ultrasonic Filed Modeling in Solid Media," DPSM-Distributed Point Source Method for Modelling Engineering Problems, edited by D. Placko and T. Kundu (Wiley, New York, 2007), pp. 143-229.

${ }^{39}$ A. Tverdokhlebov and J. Rose, "On Green's function for elastic waves in anisotropic media," J. Acoust. Soc. Am. 83, 118-121 (1988).

${ }^{40}$ C. Y. Wang and J. D. Achenbach, "Elastodynamic fundamental solutions for anisotropic solids," Geophys. J. Int. 118, 384-392 (1994).

${ }^{41} \mathrm{~V}$. K. Tewary, "Computationally efficient representation for elastostatic and elastodynamic Green's functions for anisotropic solids," Phys. Rev. B, 51, 15695-15702 (1995)

${ }^{42}$ C. Y. Wang and J. D. Achenbach, "A new method to obtain 3-D Green's functions in anisotropic solids," Wave Motion, 18, 273-289 (1993).

${ }^{43}$ G. D. Manolis, T. V. Rangelov, and P. S. Dineva, "Elastodynamic fundamental solutions for 2D inhomogeneous anisotropic domains," Proceedings of the 5th GRACM International Congress on Computational Mechanics, 2005. 